\title{
AVAILABILITY OF PERIODICALLY INSPECTED SYSTEMS WITH MARKOVIAN WEAR AND SHOCKS
}

\author{
JEFFREY P. KHAROUFEH, ${ }^{*}$ \\ DANIEL E. FINKELSTEIN ${ }^{* *}$ AND \\ DUSTIN G. MIXON, ${ }^{* *}$ Air Force Institute of Technology
}

\begin{abstract}
We analyze a periodically inspected system with hidden failures in which the rate of wear is modulated by a continuous-time Markov chain and additional damage is induced by a Poisson shock process. We explicitly derive the system's lifetime distribution and mean time to failure, as well as the limiting average availability. The main results are illustrated in two numerical examples.
\end{abstract}

Keywords: Shock model; wear process; reliability; average availability

2000 Mathematics Subject Classification: Primary 90B25

Secondary $60 \mathrm{~K} 37$

\section{Introduction}

In this paper, we consider reliability and availability measures for a single-unit system that suffers degradation due to its operating environment and the impact of shocks of random magnitude occurring at random time intervals. The system is said to experience a soft failure when its cumulative level of degradation exceeds a fixed threshold value, for example the component's nominal service life. However, it is possible that failures remain hidden until a routine (planned) inspection of the unit reveals the failure. Using a periodic inspection policy, if the unit is found to have suffered an intolerable level of degradation, it is instantaneously replaced with a new and identical unit. On the other hand, if the level of degradation observed upon inspection is acceptable (i.e. below the specified threshold value), no action is taken until the subsequent scheduled inspection. One example of a system maintained using this type of inspection policy is that of cutting equipment in manufacturing environments. Though the equipment may be operational, its cutting tool may have suffered sufficient degradation to render it ineffective in achieving engineering specifications. However, its condition may not be known until an actual measurement of the accumulated degradation is obtained. Hidden failures are also prevalent in the antennae of satellite tracking stations. In such systems the stations, situated in remote locations around the globe, may be difficult to access and inspect. Hence, periodic inspection policies are needed to ensure proper communication links with orbital satellites, to maintain such critical functions as surveillance, communications, navigation, and warning.

The impact of a random environment on reliability and availability measures has been examined extensively in the applied probability literature. Models including degradation due only to wear or only to random shocks are especially prevalent. The seminal work of

Received 4 January 2005; revision received 13 March 2006.

* Postal address: Department of Operational Sciences, Air Force Institute of Technology, Wright-Patterson Air Force Base, OH 45433-7765, USA. Email address: jeffrey.kharoufeh@afit.edu

** Postal address: Department of Mathematics and Statistics, Air Force Institute of Technology, Wright-Patterson Air Force Base, OH 45433-7765, USA. 
Esary et al. [3] has been extended in numerous directions by several authors. For instance, Çinlar [2] generalized most of the models of [3] by demonstrating that the joint process of the unit's wear level and the state of its ambient environment may be considered to be a Markov additive process, and gave several such examples. The first example considered the case when the random environment is a general Markov process with a finite state space and the wear is assumed to increase according to a Lévy process. Additionally, random shocks were assumed to occur only at environment transition epochs. The second example (see [2]) is similar to the problem discussed here, where the cumulative wear is a continuous additive functional of the operating environment, and the first time to failure is a type of first passage time for the degradation process. Although Råde [10], Shanthikumar and Sumita [11], and Nakagawa [9] provided extensions of [3], they did not incorporate the effect of the unit's operating environment on reliability measures. Igaki et al. [4], Skoulakis [12], and Kharoufeh [5] presented degradation models that explicitly incorporate the influence of the unit's operating environment but do not consider the maintenance of such systems.

More recently, other researchers have placed an emphasis on studying maintained systems. For example, Klutke et al. [8] examined the availability of an inspected system whose interinspection times and wear rates are random. Subsequently, Klutke and Yang [7] derived an availability result for a system subject to constant degradation, shocks, and a deterministic inspection policy. In a model similar to the one presented in [5] and in this paper, Kiessler et al. [6] studied the limiting average availability of a system for which the wear rate depends on a continuous-time Markov chain. Their model considers an independent, identically distributed sequence of nominal lifetimes that determine the failure criterion, rather than the deterministic threshold value we consider. However, unlike our model, that of [6] does not explicitly provide reliability measures and does not include the degradation due to random shocks.

This paper extends the results of [5] and [6] by providing both reliability and availability measures for a system subject to Markovian wear and degradation due to random shocks. Additionally, we demonstrate that the results may be implemented numerically in a straightforward manner by employing standard Laplace transform inversion algorithms. We specifically provide, in closed form, the Laplace-Stieltjes transform of the unconditional and conditional lifetime distribution functions as well as the unconditional and conditional mean system lifetimes. Using our main results, and exploiting the Markov regenerative nature of the system, we compute the limiting average availability of the system under a periodic inspection policy using the results of [6]. Moreover, we illustrate the computational ease with which the results may be obtained numerically, in two example problems.

The remainder of the paper is organized as follows. In Section 2, we provide the formal mathematical model description and notation used throughout the paper. In Section 3, we analyze the model and explicitly derive the lifetime distribution functions and the mean lifetimes as Laplace-Stieltjes transforms. In Section 4, we provide all the components needed to compute the limiting average availability of the system, and in Section 5 we provide the two illustrative numerical examples.

\section{Mathematical model}

In this section, we describe the mathematical model and notation for the maintained system with hidden failures. A single-unit system is placed into service at time 0 in perfect working order. The system accumulates degradation until a deterministic degradation threshold value, $x$, is exceeded, at which time the system is said to have failed. We denote the random time to achieve the threshold by $T_{x}$. The degradation suffered by the system is attributed to 
environment-induced wear and to shocks that occur at random time intervals. The wear- and shock-inducing mechanisms are assumed to behave independently of one another. We further describe the stochastic evolution of the system in what follows. All random variables are assumed to be defined on a complete probability space $(\Omega, \mathcal{A}, \mathrm{P})$.

The random environment is characterized by an irreducible, continuous-time Markov chain $\mathcal{Z}:=\left\{Z_{t}: t \geq 0\right\}$ on a finite state space $S:=\{1,2, \ldots, l\}$ with infinitesimal generator matrix $\boldsymbol{Q}$, transition probability functions $\pi_{i, j}(t), i, j \in S$, and initial distribution (row) vector $\boldsymbol{\alpha}$. Whenever $Z_{t}=i$, the system accumulates wear at a rate $r(i)>0, i=1,2, \ldots, l$. Define $\boldsymbol{R}_{D}:=\operatorname{diag}(r(1), r(2), \ldots, r(l))$, the $l \times l$ diagonal matrix containing the state-dependent wear rates. The total accumulated wear up to time $t$, denoted $W_{t}$, is

$$
W_{t}=\int_{0}^{t} r\left(Z_{u}\right) \mathrm{d} u .
$$

The cumulative stochastic process $\mathcal{W}:=\left\{W_{t}: t \geq 0\right\}$, commonly known as the statedependent wear process, has been studied extensively in the literature (cf. [3], [2], and [5]), independent of a shock-inducing mechanism.

In addition to environment-induced wear, the system is damaged by shocks occurring at random time intervals. The damage caused by an individual shock is assumed to be relatively small; however, the cumulative effect of small shocks may be significant, as in the case of fatigue deterioration resulting from mechanical vibrations. Denote by $N_{t}$ the number of shocks occurring up to time $t$. The corresponding counting process $\left\{N_{t}: t \geq 0\right\}$ is assumed to be a temporally homogeneous Poisson process with rate parameter $\lambda$. The damage caused by the $n$th shock is a (nonnegative) random variable $Y_{n}$ and $\left\{Y_{n}\right\}_{n=1}^{\infty}$ is an independent, identically distributed sequence with nondefective distribution function $F_{Y}(y):=\mathrm{P}\{Y \leq y\}$. The total cumulative damage attributed to shocks up to time $t$ is the random variable

$$
\beta_{t}=\sum_{n=0}^{N_{t}} Y_{n} .
$$

Therefore, the total degradation accrued by the system up to time $t$ is the sum of the degradation due to wear and that due to shocks, and is thus given by

$$
X_{t}:=W_{t}+\beta_{t}, \quad t \geq 0 .
$$

The monotonicity of $\mathcal{X}:=\left\{X_{t}: t \geq 0\right\}$ and the positivity of the degradation rates, $r(1)$, $r(2), \ldots, r(l)$, ensure the equivalence of the events $\left\{X_{t} \leq x\right\}$ and $\left\{T_{x}>t\right\}$. Thus, the system's random lifetime is given by

$$
T_{x}=\inf \left\{t>0: X_{t} \geq x\right\},
$$

namely the first time the degradation process $\mathcal{X}$ crosses level $x$. Let $G(x, t):=\mathrm{P}\left\{T_{x} \leq t\right\}$ denote the unconditional distribution function of the unit's lifetime and let its first moment be denoted by $\mathrm{E}\left[T_{x}\right]$. Note that (2) implies

$$
G(x, t)=1-\mathrm{P}\left\{X_{t} \leq x\right\} .
$$

In the remainder of the paper, we shall adopt the following notation. We let $\mathrm{E}_{i}[\cdot]=\mathrm{E}\left[\cdot \mid Z_{0}=i\right]$ and $\mathrm{P}_{i}\{\cdot\}=\mathrm{P}\left\{\cdot \mid Z_{0}=i\right\}$. Thus, in an obvious notation, the conditional distribution of the (first) lifetime is given by

$$
G_{i}(x, t)=1-\mathrm{P}_{i}\left\{X_{t} \leq x\right\} .
$$


The unconditional mean lifetime shall be denoted by $\mathrm{E}\left[T_{x}\right]$, and the conditional mean first lifetime by $\mathrm{E}_{i}\left[T_{x}\right]$. For the purpose of computing the limiting average availability of the maintained system, we obtain each of these quantities in the form of Laplace-Stieltjes transforms, in Section 3.

\section{Lifetime distribution and mean lifetime}

In this section, we provide analytical expressions for the system's lifetime distribution function and the mean system lifetime, in the form of Laplace-Stieltjes transforms (LSTs). To that end, we introduce the following definitions and notation. The complement of the lifetime distribution is

$$
R(x, t):=\bar{G}(x, t)=\mathrm{P}\left\{X_{t} \leq x\right\},
$$

which holds due to the dual relationship $T_{x}=\inf \left\{t>0: X_{t} \geq x\right\}$. Also define the joint probability distribution

$$
R_{i, j}(x, t)=\mathrm{P}\left\{X_{t} \leq x, Z_{t}=j \mid Z_{0}=i\right\}, \quad i, j \in S .
$$

Our first main result characterizes the joint distribution of the process $(\mathcal{X}, \mathcal{Z})$.

Theorem 1. The distribution function $R_{i, j}(x, t), i, j \in S$, satisfies the partial differential equation

$$
\frac{\partial R_{i, j}(x, t)}{\partial t}+\frac{\partial R_{i, j}(x, t)}{\partial x} r(j)=\lambda\left\{\left[R_{i, j}(\cdot, t) * F_{Y}\right](x)-R_{i, j}(x, t)\right\}+\sum_{k=1}^{l} q_{k, j} R_{i, k}(x, t)
$$

for $x>0$ and $t \geq 0$, where '*'denotes the convolution operator and $q_{k, j}$ are the entries of $\boldsymbol{Q}$.

Proof. We begin by considering only the environment-induced wear of the system, and then incorporate shocks. To this end, let $V_{i, j}(x, t):=\mathrm{P}\left\{W_{t} \leq x, Z_{t}=j \mid Z_{0}=i\right\}$ and let $\varepsilon>0$ denote a small time increment. Because $\mathcal{Z}$ possesses the Markov property, is temporally homogeneous, and is independent of the degradation process $\mathcal{X}$, we may write (see [5])

$$
\begin{aligned}
V_{i, j}(x, t+\varepsilon) & =\sum_{k=1}^{l} \mathrm{P}\left\{W_{t+\varepsilon} \leq x, Z_{t+\varepsilon}=j \mid Z_{t}=k, Z_{0}=i\right\} \mathrm{P}\left\{Z_{t}=k\right\} \\
& =\sum_{k=1}^{l} \mathrm{P}\left\{Z_{t+\varepsilon}=j \mid Z_{t}=k\right\} \mathrm{P}\left\{W_{t+\varepsilon} \leq x, Z_{t}=k \mid Z_{0}=i\right\} \\
& =\sum_{k=1}^{l} \pi_{k, j}(\varepsilon) V_{i, k}(x-r(k) \varepsilon, t) .
\end{aligned}
$$

Now we incorporate the impact of Poisson shocks occurring at homogeneous rate $\lambda$. The probability mass function of the number of shocks occurring up to time $\varepsilon$ is

$$
p_{n}(\varepsilon):=\mathrm{P}\left\{N_{\varepsilon}=n\right\}, \quad n \geq 0 .
$$

The magnitude of cumulative damage caused by $n$ independent shocks is given by

$$
\beta_{n}=\sum_{i=1}^{n} Y_{i}
$$


Since shock magnitudes form an independent, identically distributed sequence with distribution function $F_{Y}$, we note that

$$
\mathrm{P}\left\{\beta_{n} \leq y\right\}=: F_{Y}^{(n)}(y),
$$

the $n$-fold convolution of $F_{Y}$. By conditioning on the number of shocks in the interval $(t, t+\varepsilon)$ and the magnitude of damage due to those shocks, (5) may be replaced by

$$
R_{i, j}(x, t+\varepsilon)=\sum_{k=1}^{l} \sum_{n=0}^{\infty} \int_{0}^{\infty} \pi_{k, j}(\varepsilon) R_{i, k}(x-r(k) \varepsilon-y, t) p_{n}(\varepsilon) F_{Y}^{(n)}(\mathrm{d} y) .
$$

By substituting the appropriate expressions for $p_{0}(\varepsilon)$ and $p_{1}(\varepsilon)$ into (6) and noting that, for small $\varepsilon, \mathrm{P}\left\{N_{\varepsilon}=n\right\}=o(\varepsilon), n \geq 2$, we may write

$$
\begin{aligned}
& R_{i, j}(x, t+\varepsilon) \\
& \quad=\sum_{k=1}^{l} \pi_{k, j}(\varepsilon)\left(R_{i, k}(x-r(k) \varepsilon, t)(1-\lambda \varepsilon)+\lambda \varepsilon \int_{0}^{\infty} R_{i, k}(x-r(k) \varepsilon-y, t) F_{Y}(\mathrm{~d} y)\right) \\
& \quad+o(\varepsilon) .
\end{aligned}
$$

The transition probability functions for the $\mathcal{Z}$ process, $\pi_{i, j}(\varepsilon), i, j \in S$, can be written as

$$
\pi_{i, j}(\varepsilon)=\delta_{i, j}+\varepsilon q_{i, j}+o(\varepsilon),
$$

where $\delta_{i, j}$ assumes the value 1 when $i=j$ and the value 0 when $i \neq j$. Substituting (8) into (7) and simplifying gives

$$
\begin{aligned}
R_{i, j}(x, t+\varepsilon)= & (1-\lambda \varepsilon) R_{i, j}(x-r(j) \varepsilon, t)+\varepsilon(1-\lambda \varepsilon) \sum_{k=1}^{l} q_{k, j} R_{i, k}(x-r(k) \varepsilon, t) \\
& +\lambda \varepsilon \int_{0}^{\infty} R_{i, j}(x-r(j) \varepsilon-y, t) F_{Y}(\mathrm{~d} y) \\
& +\lambda \varepsilon^{2} \sum_{k}^{l} q_{k, j} \int_{0}^{\infty} R_{i, k}(x-r(k) \varepsilon-y, t) F_{Y}(\mathrm{~d} y)+o(\varepsilon) .
\end{aligned}
$$

Rearranging and simplifying the terms of (9), dividing through by the time increment $\varepsilon$, and taking $\varepsilon \rightarrow 0$, shows that

$$
\begin{aligned}
& \frac{\partial R_{i, j}(x, t)}{\partial t}+\frac{\partial R_{i, j}(x, t)}{\partial x} r(j) \\
& \quad=-\lambda R_{i, j}(x, t)+\sum_{k=1}^{l} q_{k, j} R_{i, k}(x, t)+\lambda \int_{0}^{\infty} R_{i, j}(x-y, t) F_{Y}(\mathrm{~d} y),
\end{aligned}
$$

where the right-most term of (10) is the convolution of the distributions $R_{i, j}$ and $F_{Y}$.

We next set out to solve the partial differential equation (4) by the method of Laplace transforms. To this end, define the $l \times l$ matrix $\boldsymbol{R}(x, t)=\left[R_{i j}(x, t)\right]$. Using matrix notation, (4) may be written as

$$
\frac{\partial \boldsymbol{R}(x, t)}{\partial t}+\frac{\partial \boldsymbol{R}(x, t)}{\partial x} \boldsymbol{R}_{D}=\lambda\left\{\left[\boldsymbol{R}(\cdot, t) * F_{Y}\right](x)-\boldsymbol{R}(x, t)\right\}+\boldsymbol{R}(x, t) \boldsymbol{Q},
$$


where $\left[\boldsymbol{R}(\cdot, t) * F_{Y}\right](x)$ denotes the convolution of each element of $\boldsymbol{R}(x, t)$ with $F_{Y}$. We next provide an LST solution, with respect to the spatial variable $x$, for the partial differential equation (11). Define the matrix LST of $\boldsymbol{R}(x, t)$ with respect to $x$ as

$$
\widetilde{\boldsymbol{R}}(u, t)=\int_{0}^{\infty} \mathrm{e}^{-u x} \boldsymbol{R}(\mathrm{d} x, t)
$$

and the $l \times l$ diagonal matrix with each element identically equal to

$$
\widetilde{F}_{Y}(u)=\int_{0}^{\infty} \mathrm{e}^{-u y} F_{Y}(\mathrm{~d} y),
$$

the LST of $F_{Y}$ with respect to $y$, as $\widetilde{\boldsymbol{F}}_{D}(u)$.

Theorem 2. The LST of the matrix $\boldsymbol{R}(x, t)$ with respect to the spatial variable $x$ is

$$
\widetilde{\boldsymbol{R}}(u, t)=\exp \left[\left(\boldsymbol{Q}+\lambda\left(\widetilde{\boldsymbol{F}}_{D}(u)-\boldsymbol{I}\right)-u \boldsymbol{R}_{D}\right) t\right],
$$

where I denotes the identity matrix.

Proof. Taking the LST of both sides of (11) with respect to $x$ yields the following first-order ordinary differential equation in $t$ :

$$
\frac{\mathrm{d} \widetilde{\boldsymbol{R}}(u, t)}{\mathrm{d} t}+\widetilde{\boldsymbol{R}}(u, t)\left(u \boldsymbol{R}_{D}+\lambda \boldsymbol{I}-\boldsymbol{Q}-\lambda \widetilde{\boldsymbol{F}}_{D}(u)\right)=\mathbf{0} .
$$

The general solution to this ordinary differential equation is obtained via the use of an integrating factor; we obtain

$$
\widetilde{\boldsymbol{R}}(u, t) \exp \left[\left(u \boldsymbol{R}_{D}+\lambda \boldsymbol{I}-\boldsymbol{Q}-\lambda \widetilde{\boldsymbol{F}}_{D}(u)\right) t\right]=\boldsymbol{\psi},
$$

where $\psi$ is a matrix of constants of integration. The final result is obtained by applying the initial condition $\widetilde{\boldsymbol{R}}(u, 0)=\boldsymbol{I}$ and rearranging terms.

The initial probability vector of the environment process $\mathcal{Z}$ is $\boldsymbol{\alpha}=\left[\alpha_{i}\right]$, with $\alpha_{i}:=$ $\mathrm{P}\left\{Z_{0}=i\right\}, i \in S$. Let $\boldsymbol{e}$ denote a column vector of 1 s and denote by $\boldsymbol{e}_{i}$ a column vector whose $i$ th element is unity and other elements are all 0 . An explicit expression for the unconditional distribution function of the system's lifetime is

$$
G(x, t)=\mathrm{P}\left\{T_{x} \leq t\right\}=1-\boldsymbol{\alpha} \boldsymbol{R}(x, t) \boldsymbol{e}
$$

and the conditional distribution is

$$
G_{i}(x, t)=\mathrm{P}_{i}\left\{T_{x} \leq t\right\}=1-\boldsymbol{e}_{i}^{\top} \boldsymbol{R}(x, t) \boldsymbol{e},
$$

where $\boldsymbol{e}_{i}^{\top}$ denotes the transpose of $\boldsymbol{e}_{i}$.

Define the LSTs of $G(x, t)$ and $G_{i}(x, t)$ with respect to $x$ as

$$
\widetilde{G}(u, t)=\int_{0}^{\infty} \mathrm{e}^{-u x} G(\mathrm{~d} x, t)
$$

and

$$
\widetilde{G}_{i}(u, t)=\int_{0}^{\infty} \mathrm{e}^{-u x} G_{i}(\mathrm{~d} x, t)
$$

respectively. 
Theorem 3. The LSTs of the unconditional and conditional first lifetime distributions, $G(x, t)$ and $G_{i}(x, t)$, with respect to $x$ are respectively

$$
\widetilde{G}(u, t)=1-\boldsymbol{\alpha} \exp \left(\left(\boldsymbol{Q}+\lambda\left(\widetilde{\boldsymbol{F}}_{D}(u)-\boldsymbol{I}\right)-u \boldsymbol{R}_{D}\right) t\right) \boldsymbol{e}
$$

and

$$
\widetilde{G}_{i}(u, t)=1-\boldsymbol{e}_{i}^{\top} \exp \left(\left(\boldsymbol{Q}+\lambda\left(\widetilde{\boldsymbol{F}}_{D}(u)-\boldsymbol{I}\right)-u \boldsymbol{R}_{D}\right) t\right) \boldsymbol{e},
$$

with $\operatorname{Re}(u)>0$.

Next we examine the unconditional and conditional mean system lifetimes, respectively $\mathrm{E}\left[T_{x}\right]$ and $\mathrm{E}_{i}\left[T_{x}\right]$, for a single-unit system subject to Markovian wear and a Poisson shock process. The LSTs of $\mathrm{E}\left[T_{x}\right]$ and $\mathrm{E}_{i}\left[T_{x}\right]$ with respect to $x$ are respectively

$$
\widetilde{\mathrm{E}}\left[T_{u}\right]=\int_{0}^{\infty} \mathrm{e}^{-u x} \mathrm{dE}\left[T_{x}\right]
$$

and

$$
\widetilde{\mathrm{E}}_{i}\left[T_{u}\right]=\int_{0}^{\infty} \mathrm{e}^{-u x} \mathrm{dE} \mathrm{E}_{i}\left[T_{x}\right] .
$$

These are obtained in the following theorem.

Theorem 4. The LST of the unconditional mean lifetime with respect to $x$ is

$$
\widetilde{\mathrm{E}}\left[T_{u}\right]=\boldsymbol{\alpha}\left(u \boldsymbol{R}_{D}-\boldsymbol{Q}-\lambda\left(\widetilde{\boldsymbol{F}}_{D}(u)-\boldsymbol{I}\right)\right)^{-1} \boldsymbol{e},
$$

and that of the conditional mean lifetime is

$$
\widetilde{\mathrm{E}}_{i}\left[T_{u}\right]=\boldsymbol{e}_{i}^{\top}\left(u \boldsymbol{R}_{D}-\boldsymbol{Q}-\lambda\left(\widetilde{\boldsymbol{F}}_{D}(u)-\boldsymbol{I}\right)\right)^{-1} \boldsymbol{e},
$$

with $\operatorname{Re}(u)>0$.

Proof. By (12), the distribution function of the system lifetime is

$$
\widetilde{G}(u, t)=1-\boldsymbol{\alpha} \exp \left[\left(\boldsymbol{Q}+\lambda\left(\widetilde{\boldsymbol{F}}_{D}(u)-\boldsymbol{I}\right)-u \boldsymbol{R}_{D}\right) t\right] \boldsymbol{e} .
$$

For brevity we write $\boldsymbol{A}=\boldsymbol{Q}+\lambda\left(\widetilde{\boldsymbol{F}}_{D}(u)-\boldsymbol{I}\right)-u \boldsymbol{R}_{D}$, to obtain the following expression for the LST of $\widetilde{G}(u, t)$ with respect to $t$ :

$$
\widetilde{G}(u, s)=\int_{0}^{\infty} \mathrm{e}^{-s t} \widetilde{G}(u, \mathrm{~d} t)=1-\boldsymbol{\alpha} \boldsymbol{A}(s \boldsymbol{I}-\boldsymbol{A})^{-1} \boldsymbol{e} .
$$

In the usual way, the mean system lifetime is obtained by evaluating the first partial derivative of $\widetilde{G}(u, s)$ with respect to $s$ at $s=0$. That is,

$$
\begin{aligned}
\widetilde{\mathrm{E}}\left[T_{u}\right] & =-\left.\frac{\partial \widetilde{G}(u, s)}{\partial s}\right|_{s=0} \\
& =-\boldsymbol{\alpha}(-\boldsymbol{A})^{-1} \boldsymbol{e} \\
& =\boldsymbol{\alpha}\left(u \boldsymbol{R}_{D}-\boldsymbol{Q}-\lambda\left(\widetilde{\boldsymbol{F}}_{D}(u)-\boldsymbol{I}\right)\right)^{-1} \boldsymbol{e} .
\end{aligned}
$$

The conditional mean lifetime is obtained analogously, by simply replacing $\widetilde{G}(u, t)$ by $\widetilde{G}_{i}(u, t)$. 


\section{Limiting average availability}

In this section, we study the asymptotic behavior of the average availability of the maintained system with hidden failures. The main result for the limiting average availability (Theorem 5) is contained in Kiessler et al. [6]; however, we review this result within the framework of our model, which differs from that of [6] in a few important ways. In our model, a failure occurs when the cumulative degradation (due to wear and shocks) reaches or exceeds a deterministic threshold. The model in [6] does not consider shocks. Moreover, the numerical approach we use for computing the limiting average availability is significantly simpler. Specifically, we apply the LSTs of Section 3 and numerical inversion to simplify the computation of the required components.

The system is inspected periodically at times in the set $I=\{k \tau: k=1,2, \ldots\}$, for some $\tau>0$. If, upon inspection, the system is found to have failed (i.e. the cumulative degradation of the system is at least as large as the tolerable level, $x$ ), then it is instantaneously replaced with a new and identical unit; however, if the system is found not to have failed, then no action is taken. Failures occurring between inspection epochs cannot be detected or repaired; thus, failures remain hidden until the next inspection epoch, and the process $\mathcal{X}$ remains at the failed level until the subsequent inspection epoch. We further assume that inspections correctly diagnose the level of degradation. Define $\Psi:=\{\psi(t): t \geq 0\}$, the right-continuous stochastic process describing the system state by

$$
\psi(t)=1\left(X_{t}<x\right)
$$

where $1(E)$ denotes the indicator function of the event $E$. As defined in [6], the system's limiting average availability is given by

$$
\bar{A}=\lim _{t \rightarrow \infty} t^{-1} \int_{0}^{t} \mathrm{E}[\psi(w)] \mathrm{d} w .
$$

Let $L_{n}$ denote the lifetime of the $n$th system and let $R_{n}$ denote the $n$th replacement epoch (with $R_{0} \equiv 0$ ). We further define $\xi_{n}=Z_{R_{n}}$, the state of the environment at the time of the $n$th replacement, and $F_{n}$, the $n$th failure epoch. The process $\left\{\xi_{n}: n \geq 0\right\}$ is an irreducible, discretetime Markov chain with one-step transition probability matrix $\boldsymbol{P}$ and stationary distribution $\boldsymbol{p}=\left[p_{i}\right], i=1,2, \ldots, l$. It is important to note that, by its nature, $\Psi=\{\psi(t): t \geq 0\}$ is not regenerative with respect to the sequence of failure or replacement epochs. This is due to the fact that successive unit lifetimes do not form an independent, identically distributed sequence of random variables unless the successive units are placed into service in identical environment states. Kiessler et al. [6] showed that the bivariate process $\left\{\left(\xi_{n}, R_{n}\right): n \geq 0\right\}$ is a Markov renewal process, and that $\{\psi(t): t \geq 0\}$ is Markov regenerative with respect to $\left\{\left(\xi_{n}, R_{n}\right): n \geq 0\right\}$. Consequently, the limiting average availability is obtained by Theorem 5 (see [6, p. 704]).

Theorem 5. The limiting average availability is given by

$$
\bar{A}=\lim _{t \rightarrow \infty} t^{-1} \int_{0}^{t} \mathrm{E}[\psi(w)] \mathrm{d} w=\frac{\sum_{i=1}^{l} p_{i} \mathrm{E}_{i}\left[F_{1}\right]}{\sum_{i=1}^{l} p_{i} \mathrm{E}_{i}\left[R_{1}\right]} .
$$

In order to compute the limiting average availability, we will obtain the transition probability matrix, $\boldsymbol{P}$, as the limit of the semi-Markov kernel of the process $\left\{\left(\xi_{n}, R_{n}\right): n \geq 0\right\}$. Subsequently, we obtain the stationary distribution, $\boldsymbol{p}$. We additionally require the conditional 
expectations $\mathrm{E}_{i}\left[R_{1}\right]$ and $\mathrm{E}_{i}\left[F_{1}\right]$. Fortunately, the LST of $\mathrm{E}_{i}\left[F_{1}\right]$ may be obtained using (14), and the conditional distribution of $F_{1}$ is given by (13). Thus, our next objective is to provide an expression for $\mathrm{E}_{i}\left[R_{1}\right]$. However, we first need to establish that $F_{1}$ is a bounded random variable. Let us re-order the wear rates so that $r_{1}<r_{2}<\cdots<r_{l}$. That is, $r_{1}$ corresponds to the minimum attainable wear rate. The following lemma holds for both the conditional and unconditional first unit lifetimes.

Lemma 1. The random variable $F_{1}$ is bounded above by $x r_{1}^{-1}$.

Proof. Let $D:=\{r(i): i=1,2, \ldots, l\}$ and let $\boldsymbol{r}:=\left(r_{1}, r_{2}, \ldots, r_{l}\right)^{\top}$ denote the column vector of ordered wear rates. Furthermore, define the set

$$
A:=\left\{\boldsymbol{a} \in \mathbb{R}_{+}^{1 \times l}: \boldsymbol{a} \boldsymbol{e}=x r_{1}^{-1}\right\},
$$

where $\mathbb{R}_{+}^{1 \times l}$ denotes the set of $l$-dimensional row vectors with strictly positive entries. We first note that

$$
\inf \{a \boldsymbol{r}+b: \boldsymbol{a} \in A, b \geq 0\} \leq x,
$$

since for $\boldsymbol{a}=x r_{1}^{-1} \boldsymbol{e}_{1}$ and $b=0$ we have $\boldsymbol{a} \boldsymbol{r}+b=x$. Moreover,

$$
\begin{aligned}
\inf \{a r+b: a \in A, b \geq 0\} & =\inf \{a r: a \in A\} \\
& \geq \inf \left\{a\left(r_{1} \boldsymbol{e}\right): \boldsymbol{a} \in A\right\} \\
& =x .
\end{aligned}
$$

From (15) and (16) we see that

$$
x=\min \{a \boldsymbol{r}+b: \boldsymbol{a} \in A, b \geq 0\} .
$$

Let $\omega \in \Omega$ and define the quantity

$$
w_{n}(\omega):=\int_{0}^{x / r_{1}} 1\left(r\left(Z_{t}(\omega)\right)=r_{n}\right) \mathrm{d} t .
$$

By summing over all possible environment states, we see that

$$
\begin{aligned}
\sum_{n=1}^{l} w_{n}(\omega) & =\sum_{n=1}^{l} \int_{0}^{x / r_{1}} 1\left(r\left(Z_{t}(\omega)\right)=r_{n}\right) \mathrm{d} t \\
& =\int_{0}^{x / r_{1}} \sum_{n=1}^{l} 1\left(r\left(Z_{t}(\omega)\right)=r_{n}\right) \mathrm{d} t \\
& =\int_{0}^{x / r_{1}} 1\left(r\left(Z_{t}(\omega)\right) \in D\right) \mathrm{d} t \\
& =\int_{0}^{x / r_{1}} \mathrm{~d} t \\
& =x r_{1}^{-1},
\end{aligned}
$$

since $r\left(Z_{t}(\omega)\right) \in D$ for almost every $t \geq 0$. It follows that the row vector $\boldsymbol{w}(\omega):=\left(w_{1}(\omega)\right.$, $\left.\ldots, w_{l}(\omega)\right)$ is a member of $A$. 
Recall that the total damage due to shocks on the interval $\left[0, x / r_{1}\right)$ is given by $\beta_{x / r_{1}}(\omega)$. Using (1), we may write

$$
\begin{aligned}
X_{x / r_{1}}(\omega) & =W_{x / r_{1}}(\omega)+\beta_{x / r_{1}}(\omega) \\
& =\int_{0}^{x / r_{1}} r\left(Z_{t}(\omega)\right) \mathrm{d} t+\beta_{x / r_{1}}(\omega) \\
& =\int_{0}^{x / r_{1}} \sum_{n=1}^{l} r_{n} 1\left(r\left(Z_{t}(\omega)=r_{n}\right)\right) \mathrm{d} t+\beta_{x / r_{1}}(\omega) \\
& =\sum_{n=1}^{l} \int_{0}^{x / r_{1}} r_{n} 1\left(r\left(Z_{t}(\omega)=r_{n}\right)\right) \mathrm{d} t+\beta_{x / r_{1}}(\omega) \\
& =\sum_{n=1}^{l} w_{n}(\omega) r_{n}+\beta_{x / r_{1}}(\omega) \\
& =\boldsymbol{w}(\omega) \boldsymbol{r}+\beta_{x / r_{1}}(\omega) .
\end{aligned}
$$

From (17) we conclude that

$$
x=\min \left\{X_{x / r_{1}}(\omega): \omega \in \Omega\right\}
$$

Since $X_{x / r_{1}}(\omega) \geq x$, it is clear that

$$
\left|F_{1}(\omega)\right|=F_{1}(\omega)=\inf \left\{t>0: X_{t}(\omega) \geq x\right\} \leq x r_{1}^{-1},
$$

i.e. that the nonnegative random variable $F_{1}$ is bounded above by $x r_{1}^{-1}$.

Lemma 1 implies that $G_{i}(x, t)=1$ for all $t \geq x r_{1}^{-1}$. Equipped with this result, we are now prepared to establish the mean of the first replacement epoch and, subsequently, the limiting average availability.

Lemma 2. The conditional expectation of the first replacement epoch is given by

$$
\mathrm{E}_{i}\left[R_{1}\right]=\tau\left(\gamma-\sum_{n=0}^{\gamma-1} G_{i}(x, n \tau)\right),
$$

where $\gamma:=\min \left\{n \geq 1: n \tau \geq x r_{1}^{-1}\right\}$ and $r_{1}:=\min \{r(i): i=1,2, \ldots, l\}$.

Proof. The first replacement time, $R_{1}$, depends explicitly on $F_{1}$ according to

$$
R_{1}=\inf \left\{w \in I: w>F_{1}\right\}
$$

By construction, $F_{1}$ corresponds to the lifetime of the first unit and, as such, has the (conditional) distribution function $G_{i}(x, t), i \in S$. By conditioning on $F_{1}$, we obtain

$$
\mathrm{E}_{i}\left[R_{1}\right]=\int_{0}^{\infty} \mathrm{E}_{i}\left[R_{1} \mid F_{1}=t\right] G_{i}(x, \mathrm{~d} t) .
$$


Because a failure remains hidden until the first inspection following the failure, the integrand of (19) is

$$
\mathrm{E}_{i}\left[R_{1} \mid F_{1}=t\right]=\left\{\begin{array}{cl}
\tau, & 0 \leq t<\tau, \\
2 \tau, & \tau \leq t<2 \tau, \\
\vdots & \\
n \tau, & (n-1) \tau \leq t<n \tau, \\
\vdots &
\end{array}\right.
$$

By substituting (20) into (19), we may write

$$
\begin{aligned}
\mathrm{E}_{i}\left[R_{1}\right] & =\tau \int_{0}^{\tau} G_{i}(x, \mathrm{~d} t)+2 \tau \int_{\tau}^{2 \tau} G_{i}(x, \mathrm{~d} t)+3 \tau \int_{2 \tau}^{3 \tau} G_{i}(x, \mathrm{~d} t)+\cdots \\
& =-\tau \sum_{n=0}^{\infty} G_{i}(x, n \tau)
\end{aligned}
$$

and by applying Lemma 1 it can be shown that the infinite series $-\sum_{n=0}^{\infty} G_{i}(x, n \tau)$ is convergent, with sum

$$
\sum_{n=0}^{\infty}-G_{i}(x, n \tau)=\gamma-\sum_{n=0}^{\gamma-1} G_{i}(x, n \tau),
$$

where $\gamma=\min \left\{n \geq 1: n \tau \geq x r_{1}^{-1}\right\}$. Therefore,

$$
\mathrm{E}_{i}\left[R_{1}\right]=\tau\left(\gamma-\sum_{n=0}^{\gamma-1} G_{i}(x, n \tau)\right) .
$$

Finally, we turn our attention to the transition probability matrix, $\boldsymbol{P}$, for the discrete-time Markov chain $\left\{\xi_{n}: n \geq 0\right\}$. Before deriving an expression for the elements of $\boldsymbol{P}$, we need to establish the conditional distribution of $R_{1}$ given the initial environment state.

Lemma 3. Assuming that the initial environment state is $i \in S$, the probability mass function of $R_{1}$ is given by

$$
\mathrm{P}_{i}\left\{R_{1}=n \tau\right\}=\Delta_{i}(x, n \tau), \quad n \geq 1,
$$

where $\Delta_{i}(x, n \tau):=G_{i}(x, n \tau)-G_{i}(x,(n-1) \tau)$ for $i \in S$ and $n \geq 1$.

Proof. Recall that $R_{1}=\inf \left\{w \in I: w>F_{1}\right\}$. Thus, we obtain the probability distribution of $R_{1}$ by conditioning on the first failure epoch given the initial state of $\mathcal{Z}$. For $n \geq 1$,

$$
\begin{aligned}
\mathrm{P}_{i}\left\{R_{1}=n \tau\right\} & =\int_{0}^{\infty} \mathrm{P}_{i}\left\{R_{1}=n \tau \mid F_{1}=t\right\} G_{i}(x, \mathrm{~d} t) \\
& =\int_{0}^{\infty} 1(t \in[(n-1) \tau, n \tau]) G_{i}(x, \mathrm{~d} t) \\
& =\int_{(n-1) \tau}^{n \tau} G_{i}(x, \mathrm{~d} t) \\
& =G_{i}(x, n \tau)-G_{i}(x,(n-1) \tau) .
\end{aligned}
$$


Finally, we obtain the one-step transition probability matrix for $\left\{\xi_{n}: n \geq 0\right\}$ by computing the limit of the semi-Markov kernel matrix of the Markov renewal process $\left\{\left(\xi_{n}, R_{n}\right): n \geq 0\right\}$.

Theorem 6. The $(i, k)$ th element of the transition probability matrix $\boldsymbol{P}=\left[p_{i, k}\right], i, k \in S$, is given by

$$
p_{i, k}=\sum_{n=1}^{\gamma} \pi_{i, k}(n \tau) \Delta_{i}(x, n \tau),
$$

where $\Delta_{i}(x, n \tau)$ is obtained from

$$
\Delta_{i}(x, n \tau)=\mathcal{L}^{-1}\left\{u^{-1}\left[\widetilde{G}_{i}(u, n \tau)-\widetilde{G}_{i}(u,(n-1) \tau)\right]\right\},
$$

$\mathcal{L}^{-1}$ denoting the inverse Laplace transform operator. The integer $\gamma$ is as defined in Lemma 2.

Proof. Let $K_{i, k}(t):=\mathrm{P}\left\{\xi_{1}=k, R_{1} \leq t \mid \xi_{0}=i\right\}$ denote the $(i, k)$ th element of the semiMarkov kernel, $\boldsymbol{K}(t)$, of the Markov renewal process $\left\{\left(\xi_{n}, R_{n}\right): n \geq 0\right\}$. Let us further define

$$
\widetilde{K}_{i, k}(s)=\mathrm{E}_{i}\left[\mathrm{e}^{-s R_{1}} 1\left(Z_{R_{1}}=k\right)\right],
$$

with $\operatorname{Re}(s)>0$. By conditioning on $R_{1}$, we may write

$$
\begin{aligned}
\mathrm{E}_{i}\left[\mathrm{e}^{-s R_{1}} 1\left(Z_{R_{1}}=k\right)\right] & =\sum_{n=1}^{\infty} \mathrm{E}_{i}\left[\mathrm{e}^{-s R_{1}} 1\left(Z_{R_{1}}=k\right) \mid R_{1}=n \tau\right] \mathrm{P}_{i}\left\{R_{1}=n \tau\right\} \\
& =\sum_{n=1}^{\infty} \mathrm{e}^{-s n \tau} \mathrm{E}_{i}\left[1\left(Z_{n \tau}=k\right)\right] \Delta_{i}(x, n \tau) \\
& =\sum_{n=1}^{\infty} \mathrm{e}^{-s n \tau} \mathrm{P}_{i}\left\{Z_{n \tau}=k\right\} \Delta_{i}(x, n \tau) \\
& =\sum_{n=1}^{\infty} \mathrm{e}^{-s n \tau} \pi_{i, k}(n \tau) \Delta_{i}(x, n \tau) .
\end{aligned}
$$

By the final value theorem of Laplace transforms, we may obtain $p_{i, k}$ as

$$
p_{i, k}=\lim _{t \rightarrow \infty} K_{i, k}(t)=\lim _{s \rightarrow 0} \widetilde{K}_{i, k}(s)=\lim _{s \rightarrow 0} \sum_{n=1}^{\infty} \mathrm{e}^{-s n \tau} \pi_{i, k}(n \tau) \Delta_{i}(x, n \tau) .
$$

Recall from Lemma 1 that $\Delta_{i}(x, n \tau)=0$ for all $n \geq \gamma+1$; we thus have

$$
\begin{aligned}
p_{i, k} & =\lim _{s \rightarrow 0} \sum_{n=1}^{\infty} \mathrm{e}^{-s n \tau} \pi_{i, k}(n \tau) \Delta_{i}(x, n \tau) \\
& =\lim _{s \rightarrow 0} \sum_{n=1}^{\gamma} \mathrm{e}^{-s n \tau} \pi_{i, k}(n \tau) \Delta_{i}(x, n \tau) \\
& =\sum_{n=1}^{\gamma} \lim _{s \rightarrow 0} \mathrm{e}^{-s n \tau} \pi_{i, k}(n \tau) \Delta_{i}(x, n \tau) \\
& =\sum_{n=1}^{\gamma} \pi_{i, k}(n \tau) \Delta_{i}(x, n \tau) .
\end{aligned}
$$


In comparing (22) with Corollary 1 of [6], we note that our expression requires the evaluation of only a finite sum and (numerical) Laplace transform inversion to obtain $\Delta_{i}(x, n \tau), \mathrm{E}_{i}\left[F_{1}\right]$, and $\mathrm{E}_{i}\left[R_{1}\right]$. Fortunately, numerical inversion algorithms abound for this task, and the truncation point $\gamma$ is well defined by the parameters $\tau$ and $r_{1}$. By contrast, the results of [6] require evaluation of an infinite Fourier series to compute $p_{i, k}$ and the conditional expectations.

\section{Numerical examples}

In this section, we illustrate the computation of the limiting average availability by means of two examples. In each example, we compute the conditional expectations $\mathrm{E}_{i}\left[F_{1}\right]$ and $\mathrm{E}_{i}\left[R_{1}\right]$, the matrix $\boldsymbol{P}$, the stationary distribution, $\boldsymbol{p}$, and the limiting average availability, $\bar{A}$. The results were obtained via numerical Laplace transform inversion using the robust technique of [1]. The algorithm was coded and executed in the MATLAB ${ }^{\circledR}$ computing environment. In each of the two cases, $\mathcal{Z}$ is an irreducible, continuous-time Markov chain on finite state space $S$ with generator matrix $\boldsymbol{Q}$.

Example 1. $(l=2$.) The first case is a simple environment which toggles between two distinct states $(S=\{1,2\})$ according to the generator matrix

$$
\boldsymbol{Q}=\left[\begin{array}{rr}
-\frac{25}{3} & \frac{25}{3} \\
\frac{25}{3} & -\frac{25}{3}
\end{array}\right] \text {. }
$$

The diagonal matrix of state-dependent wear rates is

$$
\boldsymbol{R}_{D}=\operatorname{diag}\left(\frac{13}{12}, \frac{1}{4}\right),
$$

while the LST of the shock magnitude distribution function is

$$
\widetilde{F}_{Y}(u)=\frac{4}{4+u} .
$$

Shocks occur according to a Poisson process with rate parameter $\lambda=0.5$. The threshold degradation level for failure, in units of degradation, is $x=1$. Inspections occur every $\tau=0.1$ time units. By applying equations (14) and (18), we compute

$$
\mathrm{E}_{1}\left[F_{1}\right]=1.2976, \quad \mathrm{E}_{2}\left[F_{1}\right]=1.3609
$$

and

$$
\mathrm{E}_{1}\left[R_{1}\right]=1.3475, \quad \mathrm{E}_{2}\left[R_{1}\right]=1.4109 .
$$

By selecting the truncation point $\gamma$ using Theorem 6 , the transition probability matrix is found to be

$$
\boldsymbol{P}=\left[\begin{array}{ll}
0.5002 & 0.4998 \\
0.4998 & 0.5002
\end{array}\right]
$$

with the obvious stationary distribution

$$
\boldsymbol{p}=\left[\begin{array}{ll}
0.5000 & 0.5000
\end{array}\right]
$$

Subsequently, the limiting average availability is found to be

$$
\bar{A}=\frac{\sum_{i=1}^{2} p_{i} \mathrm{E}_{i}\left[F_{1}\right]}{\sum_{i=1}^{2} p_{i} \mathrm{E}_{i}\left[R_{1}\right]}=0.9638 .
$$


TABLE 1: Stationary probabilities and conditional means (Example 2).

\begin{tabular}{crrrrr}
\hline Measure & \multicolumn{1}{c}{$i=1$} & \multicolumn{1}{c}{$i=2$} & \multicolumn{1}{c}{$i=3$} & \multicolumn{1}{c}{$i=4$} & \multicolumn{1}{c}{$i=5$} \\
\hline$p_{i}$ & 0.1160 & 0.0277 & 0.5308 & 0.3135 & 0.0120 \\
$\mathrm{E}_{i}\left[R_{1}\right]$ & 11.6861 & 11.4400 & 11.4752 & 11.2014 & 11.3457 \\
$\mathrm{E}_{i}\left[F_{1}\right]$ & 9.1931 & 8.9485 & 8.9836 & 8.7116 & 8.8542 \\
\hline
\end{tabular}

Example 2. $(l=5$.) In this case, the environment process is a five-state, continuous-time Markov chain with state space $S=\{1,2,3,4,5\}$. The generator matrix for the process is

$$
\boldsymbol{Q}=\left[\begin{array}{rrrrr}
-0.500 & 0.125 & 0.125 & 0.125 & 0.125 \\
0.400 & -2.000 & 0.400 & 0.600 & 0.600 \\
0.025 & 0.025 & -0.100 & 0.025 & 0.025 \\
0.050 & 0.050 & 0.050 & -0.200 & 0.050 \\
1.500 & 1.000 & 1.000 & 1.500 & -5.000
\end{array}\right]
$$

The diagonal matrix of state-dependent wear rates is

$$
\boldsymbol{R}_{D}=\operatorname{diag}(1,2,3,4,10),
$$

while the LST of the shock magnitude distribution function is

$$
\widetilde{F}_{Y}(u)=\left(\frac{0.2}{0.2+u}\right)^{8} .
$$

Shocks occur according to a Poisson process with parameter $\lambda=0.25$. The threshold degradation level for failure of the system, in units of degradation, is $x=100$. Inspections occur every $\tau=5$ time units. Applying (22) gives

$$
\boldsymbol{P}=\left[\begin{array}{lllll}
0.1393 & 0.0300 & 0.4810 & 0.3368 & 0.0129 \\
0.1262 & 0.0291 & 0.4868 & 0.3454 & 0.0126 \\
0.1045 & 0.0258 & 0.6115 & 0.2471 & 0.0112 \\
0.1255 & 0.0301 & 0.4181 & 0.4133 & 0.0130 \\
0.1268 & 0.0291 & 0.4876 & 0.3439 & 0.0126
\end{array}\right] .
$$

Table 1 summarizes the stationary probabilities and the conditional expectations for this case.

From these values, we compute the limiting average availability to be

$$
\bar{A}=\frac{\sum_{i=1}^{5} p_{i} \mathrm{E}_{i}\left[F_{1}\right]}{\sum_{i=1}^{5} p_{i} \mathrm{E}_{i}\left[R_{1}\right]}=0.7817 .
$$

\section{Acknowledgements}

We graciously acknowledge the constructive comments of an anonymous referee. This research was supported by the US Air Force Office of Scientific Research under agreement QAF185045200004. The views expressed in this paper are those of the authors and do not reflect the official policy or position of the United States Air Force, Department of Defense, or the US Government. 


\section{References}

[1] Aвате, J. And Whitt, W. (1995). Numerical inversion of Laplace transforms of probability distributions. ORSA J. Comput. 7, 36-43.

[2] ÇInLAR, E. (1977). Shock and wear models and Markov additive processes. In Theory and Application of Reliability: with Emphasis on Bayesian and Nonparametric Methods, eds I. N. Shimi and C. P. Tsokos, Academic Press, New York, pp. 193-214.

[3] Esary, J. D., Marshall, A. W. and Proschan, F. (1973). Shock models and wear processes. Ann. Prob. 1, 627-649.

[4] Igaki, N., Sumita, U. and Kowada, M. (1995). Analysis of Markov renewal shock models. J. Appl. Prob. 32, 821-831.

[5] Kharoufeh, J. P. (2003). Explicit results for wear processes in a Markovian environment. Operat. Res. Lett. 31, 237-244.

[6] Kiessler, P. C., Klutke, G.-A. And YAng, Y. (2002). Availability of periodically inspected systems subject to Markovian degradation. J. Appl. Prob. 39, 700-711.

[7] Klutke, G.-A. And Yang, Y. (2002). The availability of inspected systems subject to shocks and graceful degradation. IEEE Trans. Reliab. 51, 371-374.

[8] Klutke, G.-A., Wortman, M. and Ayhan, H. (1996). The availability of inspected systems subject to random deterioration. Prob. Eng. Inf. Sci. 10, 109-118.

[9] Nakagawa, T. (1979). Replacement problem of a parallel system in random environment. J. Appl. Prob. 16, 203-205.

[10] RÅDE, J. (1976). Reliability systems in random environment. J. Appl. Prob. 13, 407-410.

[11] Shanthikumar, J. G. and Sumita, U. (1983). General shock models associated with correlated renewal sequences. J. Appl. Prob. 20, 600-614.

[12] Skoulakis, G. (2000). A general shock model for a reliability system. J. Appl. Prob. 37, 925-935. 